\title{
Drawing Assist System for Cerebral Palsy Patients Considering the Involuntary Movements
}

\author{
Tomoyuki Nakao, Hirokazu Matsui, Ken'ichi Yano, Naruto Miyagawa, Naoki Kubota and Satoshi Horihata
}

\begin{abstract}
Creative activities, such as painting and playing instruments, are sources of satisfaction and fulfilment for people with disabilities. However, some individuals with disabilities cannot satisfactorily enjoy such activities because of involuntary movements. In this study, we developed a drawing assist system for users with athetoid cerebral palsy and muscle tension, who experience involuntary movement. To date, assist systems have had difficulty in distinguishing involuntary movement from voluntary movement, and thus we designed a position correction filter to attenuate involuntary movement on the basis of the characteristics of the magnitude of velocity with respect to involuntary movement. Our system enabled drawing based on the user's own senses and motor control, even when involuntary movement occurred.
\end{abstract}

\section{INTRODUCTION}

Many people with physical disabilities enjoy creative activities as a means of self-express

ion and motivation in life. Painting is one such activity that the physically disabled can perform using residual functions. For instance, one can paint by using hand dexterity if arm function is limited, or by using the mouth or lower limb if the function of both upper limbs is limited. In recent years, the development of assistive equipment and interactive software has brought computer-based painting to people with disabilities. Whether the act of painting can be satisfactorily enjoyed depends on the degree of the disability. Insofar as involuntary movements affect body control, precise finemotor movements become difficult to perform, limiting the ability to perform desired tasks such as painting.

To address these problems for people with involuntary movements who draw using computer graphics, studies have been conducted on systems that filter the effects of involuntary movements from the input signal of a device so that the on-screen pointer does not show unintended movement. IBM developed the Assistive Mouse Adapter by using a simple design where the cut-off frequency of the linear low-pass filter is tuned with a dial [1]. Some methods that cut off a frequency or frequency band are favorable for periodic involuntary movements that have a characteristic frequency, such as the tremor in Parkinson' $\mathrm{s}$ disease. In other work, many studies have been conducted

This study was supported in part by city area grants from the southern Gifu innovation cluster program.

T. Nakao, H. Matsui and K. Yano are with the Department of Mechanical Engineering, Mie University 1577 Kurimamachiya-cho, Tsu City, Mie 5148507, Japan nakao@robot.mach.mie-u.ac.jp

N. Miyagawa and N. Kubota are with the Research and Development Division, Gifu Prefecture, Research Institute for Human Life Technology 1154, Yamada, Takayama 506-0058, Japan

S. Horihata is with the School of Dentistry, Nihon University, 2-870-1 Matsudo, Chiba 501-1193, Japan using the moving average method for nonperiodic involuntary movements, such as those seen in athetosis. Morimoto developed a painting tool based on the moving average method that attenuates the effects of involuntary movements in real time, and proposed the averaging switching and compulsory compensation methods as solutions for attenuating the effects of sudden shaking[2]. Shiraishi proposed a linesmoothing method that applies the additional moving average method to a distance between an input termination point and a termination point corrected by another moving average method[3]. These methods are favorable for users with partial paralysis, who can move and click a mouse on a table. For users with severe spasticity that makes it difficult to keep the input device on a plane, the irregularity of the involuntary movement and its variable strength should be considered. Our research group has proposed a method that changes attenuation based on the strength of involuntary movements by using the magnitude of velocity from a three-dimensional input device[4]. However, a method based on the moving average method needs to be designed with a long moving average period enough to determine the attenuation strength, which worsens operability and limits what the user may draw. Against this background, a drawing assist system that attenuates nonperiodic involuntary movements and exhibits superior operability is desired.

The purpose of this study is to analyze the involuntary movements of a user with athetoid cerebral palsy during drawing manipulation, to design a position correction filter for attenuating the effects of involuntary movements in real time, and to develop a drawing assist system that enables free-form drawing, even in the presence of involuntary movements.

\section{Drawing Assist System for Patients with Cerebral Palsy}

\section{A. Cerebral Palsy}

Cerebral palsy is a disease characterized by involuntary movements; the condition results from damage to the motor control centers of the brain before, during, or shortly after birth [5]. In individuals with cerebral palsy a common symptom is athetosis, which is characterized by involuntary movement and low muscle tone. Muscle tone fluctuates with voluntary movement and mental distress, which makes it difficult for the patient to achieve continuous postural maintenance. These excessive movements do not stop occurring when voluntary movements are attempted[6]. Nakai reported on the results of analyzing power grip movement, and extension and flexion of the wrist, in patients with cerebral palsy; 
it was found that motor control in patients with cerebral palsy is less developed compared with unaffected individuals, and that the motor skills of patients with athetosis are less developed than those of patients with spasticity in terms of speed, timing, joint angle, and strength. Strong hypertonic behavior in extensor muscle groups, imbalance between flexor and extensor muscle groups, instantaneous movements, surges of power, and difficulty of power control are all characteristics of athetosis[7]. In particular, the involuntary movements of individuals with athetoid cerebral palsy and muscle tension occur suddenly and are considerably heightened by exertion. These involuntary movements manifest themselves when a person with the condition attempts to maintain posture or sustain a certain movement; in addition, the muscle tone of people with athetoid cerebral palsy increases during postural maintenance and decreases while at rest [8]. There are over 60,000 people in Japan with cerebral palsy, characterized by generalized impaired motor function [9][10].

\section{B. Experimental setup}

The experimental setup of this study is shown in Fig. 1. The input device is located in front of the user and inclined at an angle of 35 [deg] to fit the user's posture. The display is located in front of the user in an easy-tosee position. When considering an input device for users who experience severe involuntary movements, a mouse and graphics tablet are unsuitable because they require planar postural maintenance; instead, a device that can move in three dimensions and measure three-dimensional coordinates is required. An additional concern is that the users can sometimes involuntarily loses their grip on an object; for example, if a user was to release and drop a graphics tablet, it would be almost impossible for it to be retrieved without assistance. From these considerations, a compact PHANToM Omni (SensAble, Inc.) haptic interface [11] is used as an input device. The point of rotation at the center of the gimbal is used as the input point $P$ for drawing. The input coordinate system $\Sigma_{P}\left(x_{P}, y_{P}, z_{P}\right)$ is based on the input device and defined so that right and left are along the $\mathrm{x}$-axis, front and back are along the y-axis, and up and down are along the z-axis. Fig. 1 shows the input point $P$ of the input device; the arrows indicate the positive direction of each axis. The plane used for drawing in $\Sigma_{P}$ is defined as $x_{P} y_{P}$, the angle of which is sloped by the orientation of the input device. The origin of the two-dimensional drawing coordinate system $\Sigma_{D}\left(x_{D}, y_{D}\right)$ on the display is defined in the lower left
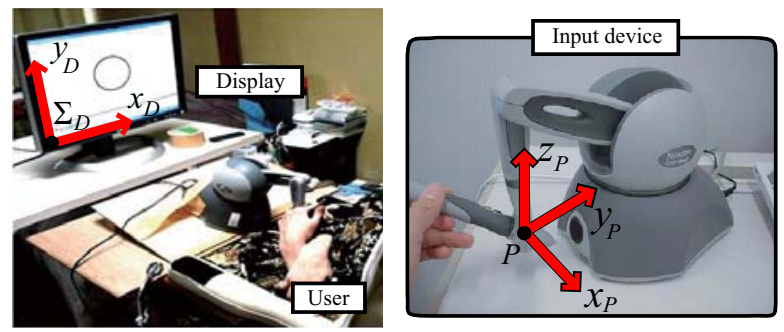

Fig. 1. Experimental setup for drawing assist system corner. Positive directions correspond for $x_{P}$ and $x_{D}$, and for $y_{P}$ and $y_{D}$.

The involuntary movements in athetosis make it difficult to manipulate any buttons and objects with both upper limbs. Thus drawing judgments must be based on only stylus manipulation. Another goal of this system is intuitive operability such as with an actual brush and canvas. An upswing and a downswing manipulation with the stylus are used for drawing judgement criteria. The drawing start criterion is designed, as shown in Equation (1), such that drawing starts when the difference between the current $\mathrm{z}$ coordinate and the $\mathrm{z}$-coordinate from 0.50 [s] before exceeds $0.05[\mathrm{~m}]$. The drawing end condition is designed, as shown in (2), such that the drawing ends when the difference between the current $\mathrm{z}$-coordinate and the z-coordinate from 0.19 [s] before exceeds $0.03[\mathrm{~m}]$. After receiving informed consent from the participant, a video camera was set up at the angle of Fig. 1 to check the correspondence between the participant's actual movements and the measured movements. The raster graphics editor used in this study was ArtRage version 2.5 (e frontier, Ltd.)[12]. The color select and undo functions were handled with a palette and a button on the window that worked with the input device.

$$
\begin{gathered}
\Delta z_{P}=z_{P}(t)-z_{P}(t-0.5) \leq-0.05 \quad[\mathrm{~m}] \\
\Delta z_{P}=z_{P}(t)-z_{P}(t-0.19) \geq 0.03 \quad[\mathrm{~m}]
\end{gathered}
$$

\section{ANALYSIS OF INVOLUNTARY MOVEMENTS DURING DRAWING}

We conducted experiments to investigate how involuntary movements influence movements during drawing. A participant traced a design, in this case an ogee (a shape similar to an "S"). The ogee design allows us to evaluate whether a participant has the required manipulation capabilities to represent a shallow curve whose direction continuously changes and to confine their drawing to the designated boundaries. The participant was instructed to begin drawing in the right square part of the ogee and sustain the drawing motion through the curved section before finally ending the drawing in the left circular part.

Fig. 2 shows the experimental results. All three examples in Fig. 2 show that it is difficult for the participant to perform the curve tracing and to start and end the drawing at the designated positions.

From these results, involuntary movements cause the participant to lose control of voluntary movements; consequently, the participant encounters difficulty in performing

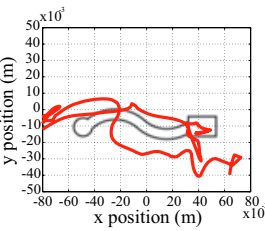

(a)

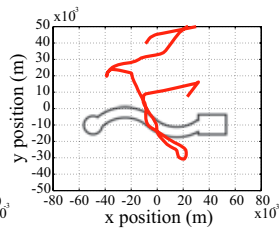

(b)

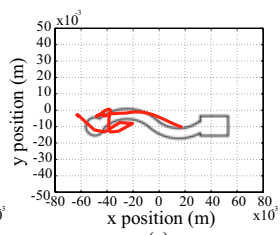

(c)
Fig. 2. Tracing results of raw data on $x-y$ plane 
the manipulations required to trace a shape whose direction continuously changes as in typical graphical elements.

The severity of athetosis varies, but individuals who experience strong involuntary movements almost completely lose complete control of voluntary movements. Fig. 3 shows a trajectory on the xy-plane generated by a participant with strong involuntary movements during a 2.5 [s] span in the ogee tracing experiment mentioned above. The participant has generated a form that is wider than the intended shape and exhibits irregular direction, distance, and frequent veer. Considering the characteristics of the involuntary movements mentioned in Section 2, each velocity component contributes to (3), the magnitude of velocity, which is used as an indicator of the strength of involuntary movements[4].

$$
V_{s}=\sqrt{v_{p x}^{2}+v_{p y}^{2}+v_{p z}^{2}}
$$

The change of the velocity between weak and strong involuntary movements is shown in Fig. 4. A histogram of the velocity is shown in Fig. 5. Examples (a) through (c) in Figs. 4 and 5 correspond to cases (a) through (c) in Fig. 2 showing drawing attempts that include weak involuntary movements. Example (d) in Figs. 4 and 5 corresponds to the data on the strong involuntary movements shown in Fig. 3.

From the distribution results of Fig. 5, we can see from the data above that weak and strong involuntary movements differ greatly in terms of the velocity frequencies; strong involuntary movements most frequently occur at the velocities greater than $0.20[\mathrm{~m} / \mathrm{s}]$.

\section{Adaptive InVoluntary Behavior AtTenuation FILTER}

From the involuntary movement analysis results above, a position control filter is required to prevent the display pointer from overrunning the target when a user experiences involuntary movement by attenuating the effects of involuntary movements and adapting to the changes in velocity. Additionally, the attenuation characteristics of the filter should be based on the involuntary movements and manipulation characteristics of individuals, not the subjective views of a researcher or simple trial-and-error methods. We designed

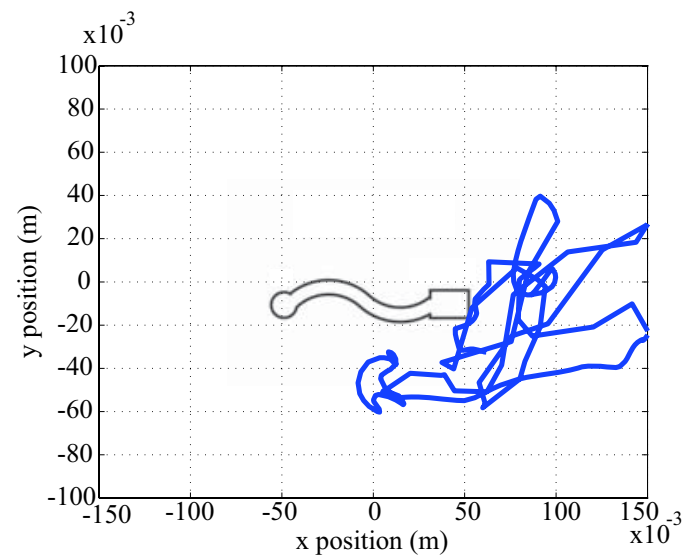

Fig. 3. Behavior of strong involuntary movement on $x-y$ plane
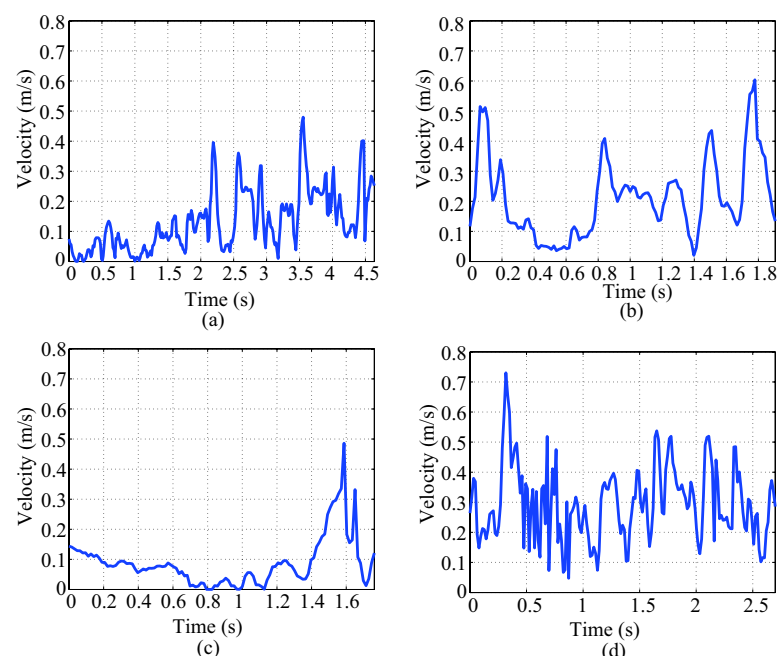

Fig. 4. Change of the magnitude of velocity
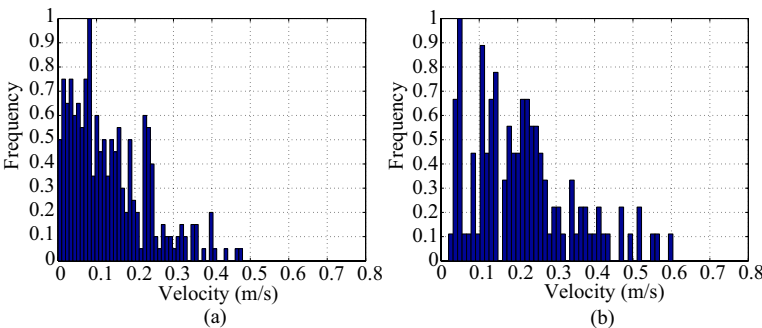

(b)

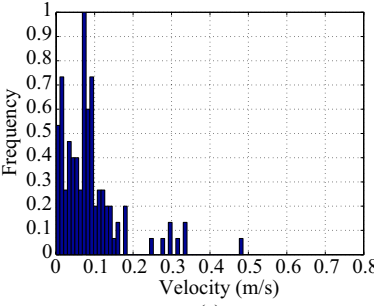

(c)

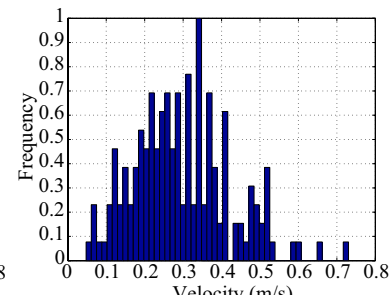

(d)

Fig. 5. Histogram of the magnitude of velocity with respect to involuntary movements

the adaptive involuntary behavior attenuation filter (AIBAF) to satisfy these requirements. Fig. 6 shows the AIBAF block diagram. The coordinates of the corrected point at sample $i$, $G(i)=\left(x_{G}(i), y_{G}(i), z_{G}(i)\right)$, as shown in (4), is updated by using a coordinate of the corrected point before a sample $G(i-1)$, the current measurement of the coordinate of input point $P(i)$, and an attenuation weight coefficient $w(i)$. The range of $w(i)$ is $0<w(i) \leq 1$. The priority of $P(i)$ becomes higher when $w(i)$ is close to 1 ; the priority of $G(i-1)$ becomes higher when $w(i)$ is close to 0 . This filter with suitably low $w(i)$ can attenuate involuntary excessive movement and make the pointer move slower. The following equations are used to compute each component of the $x-, y-$ and $\mathrm{z}$-coordinates.

$$
G(i)=(1-w(i)) G(i-1)+w(i) P(i)
$$

The attenuation weight coefficient $w(i)$, given by (5), is designed as an exponential function with respect to the 


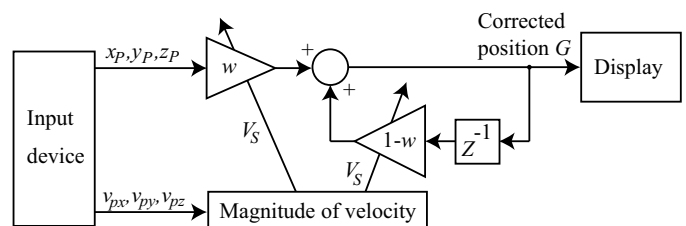

Fig. 6. Block diagram of adaptive involuntary behavior attenuation filter

velocity $V_{s}(i)$ given by (3). An upper limit coefficient $\alpha_{u l}$, in (5), is a design parameter used to set the maximum value of $w(i)$. The range of $\alpha_{u l}$ is $0<\alpha_{u l}<1$. $\alpha_{u l}$ was set to 0.003 to ensure operability during manipulation, except for during the drawing process. The attenuation weight coefficient $w(i)$ stays near $\alpha_{u l}$ at low velocities characteristic of weak involuntary movements, and decreases as the value of the velocity rises, and becomes nearly 0 at high velocity values characteristic of strong involuntary movements. $v_{t h}$ is defined as a transition velocity between weak and strong involuntary movements. A transition attenuation $D_{t h}$ is a parameter that controls the value of the attenuation weight coefficient $w(i)$ when the velocity $V_{s}(i)$ equals the transition velocity $v_{t h}$. Thus, $w(i)=\alpha_{u l} D_{t h}$ when $V_{s}(i)=v_{t h}$ and the attenuation enhancement coefficient $\beta(i)$, discussed below, is 1 . The range of $D_{t h}$ is $0<D_{t h}<1$. The change in $w(i)$ is gradual as $D_{t h}$ approaches 1 ; it asymptotically approaches 0 with a steep gradient as $D_{t h}$ approaches 0 . Thus we can use $D_{t h}$ to change the behavior of $w(i)$. The transition velocity $v_{t h}$ was set to $0.20[\mathrm{~m} / \mathrm{s}]$. The transition attenuation $D_{t h}$ was set to 0.11 .

$$
w(i)=\alpha_{u l} \beta(i) D_{t h}^{\frac{V_{s}(i)}{v_{t h}}}
$$

$\beta(i)$ is a coefficient to enhance attenuance and position correction. Drawing judgment velocity, $V_{D}(i)$, from the relation above is designed according to (6) by using the difference between $\mathrm{z}$ - and $\mathrm{x}$-axis speeds, $\left|v_{p z}(i)\right|-\left|v_{p x}(i)\right|$, and the difference between z- and y-axis speeds, $\left|v_{p z}(i)\right|-\left|v_{p y}(i)\right|$. When $V_{D}(i)>0$ is satisfied, the value of $\beta(i)$ changes based on drawing judgment velocity $V_{D}(i)$, as shown in (7). We can change the behavior of $\beta(i)$ by using $D_{\beta}$ and $v_{\beta}$, similar to the relation that $D_{t h}$ and $v_{t h}$ have with $w(i)$. Additionally, $\beta(i)=1$ under the condition $V_{D}(i) \leq 0$, and even if $V_{D}(i)>0$ when the $\mathrm{x}$ and $\mathrm{y}$-axis velocities are high, $\beta(i)$ stays near 1 , and thus drawing is not hindered. $D_{\beta}$ is designed in the way that $w(i)$ becomes close to zero when $v_{\beta}=V_{D}(i)$. $D_{\beta}$ was set to 0.01 and $v_{\beta}$ was set to $1.00[\mathrm{~m} / \mathrm{s}]$.

$$
\begin{gathered}
V_{D}(i)=2\left|v_{p z}(i)\right|-\left|v_{p x}(i)\right|-\left|v_{p y}(i)\right| \\
\beta(i)=D_{\beta}^{\frac{V_{D}(i)}{v_{\beta}}}
\end{gathered}
$$

\section{Drawing Assist EXPERIMENT}

We carried out a drawing assist experiment with AIBAF. The drawing assist experiment task was to trace an ogee, similar to the experiments above, and to trace the outline of a flower design.

The experimental results of ogee tracing are shown in Fig. 7. Figures (a) through (c) compare the trajectories of the raw data and the AIBAF-corrected data for the three tracings performed in the task; figure (d) shows the results of the drawing using the raster graphics editor.

In Fig. 7(a) through (c) the trajectories of the raw data are outside than the tracing design, while the start and end of the drawing trajectory corrected by AIBAF is within the designated boundaries; additionally, most curve tracing is stable and within the curve frame. The results in Fig. 7(a) and (b) show that the corrected trajectory strayed toward the upper side of the display before reaching the end point through purposeful operation. Next we will discuss the experimental results for correcting the drawing start and end positions. With respect to the manipulations in Fig. 7(a), the change in the absolute value of each velocity component, the drawing judgment velocity $V_{D}(i)$, and the attenuation weight coefficient $w(i)$ during a $5[\mathrm{~s}]$ span that includes the drawing start movement are shown in Fig. 8; the change with respect to the drawing end movement is similarly shown in Fig. 9. In these figures, the instance that satisfies (1) and (2) is shown as a point, and the elapsed time of the condition is marked with a dotted line. We see $w(i)$ decreases as $V_{D}(i)$ increases. The $w(i)$ value notably decreases and stays near zero during and around the time span when involuntary movement occur. The value of $V_{D}(i)$ is low during movements based on drawing, and thus $\beta(i)$ does not appear to hinder drawing of graphical elements. Strong involuntary movements did not occur in this experiments.

The next task was to trace the outline of a plant and consider the design of actual painting. Specifically, this task involved tracing the outline of a watermarked example of a tulip (Fig. 10 (a)). This example was selected by the participant from ten options of various plants and designs in

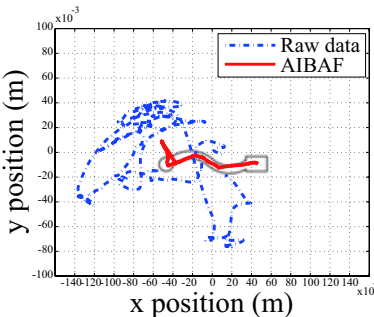

(a)

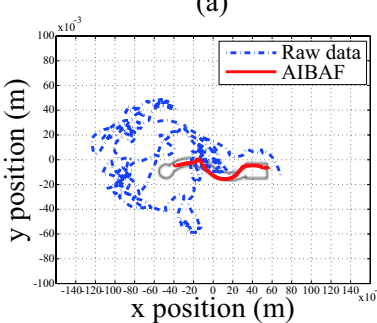

(c)

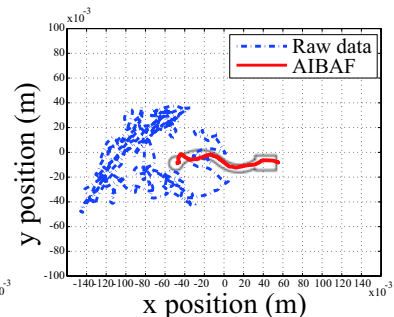

(b)

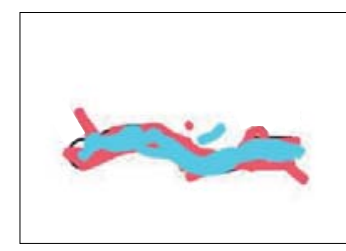

(d)
Fig. 7. AIBAF drawing results and comparison of trajectories on the xyplane 

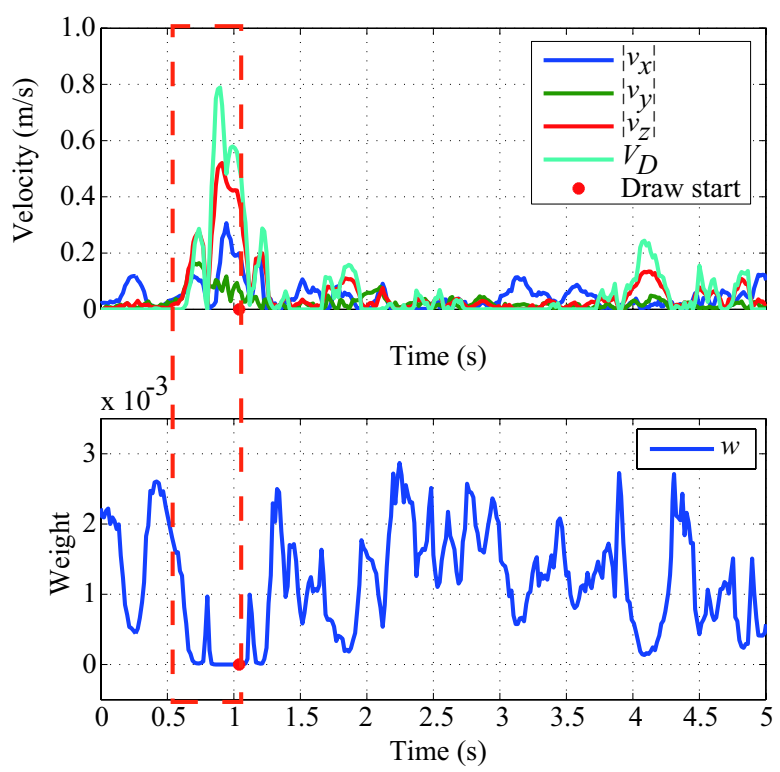

Fig. 8. Change of velocities and attenuation weight at draw start

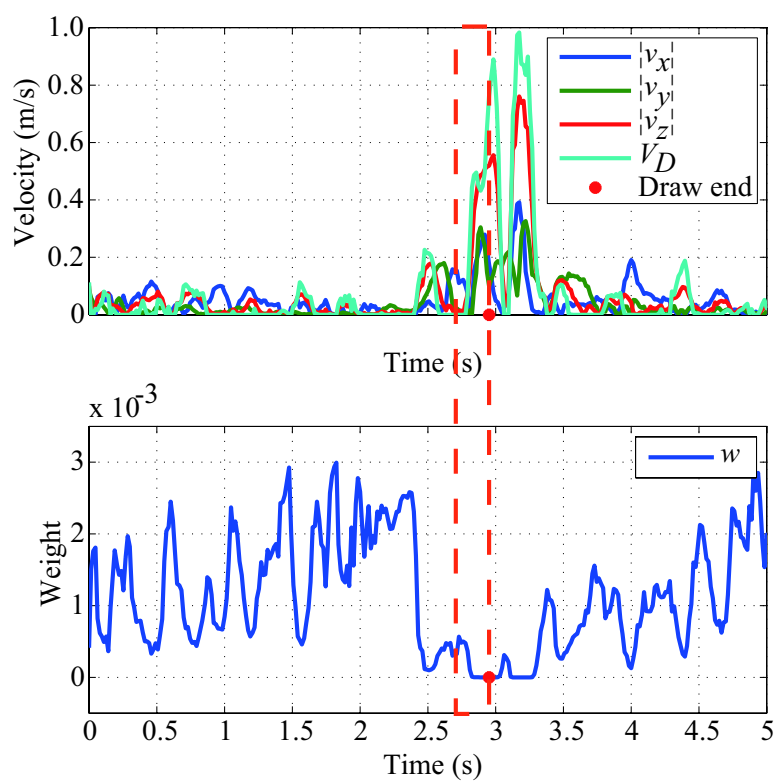

Fig. 9. Change of velocities and attenuation weight at draw end

photographs to illustrations. The raw drawing data are shown in Fig. 10 (b). The participant was able to perform relevant manipulations, but lost enthusiasm to achieve the task when about 38 [s] had passed. The AIBAF-corrected drawing is shown in Fig. 10 (c). We see that the participant was able to draw a picture similar to the example. The time required to finish the task was about 35 [min] with no interruptions. The color change and undo functions were controlled by the participant using functions on the paint tool without having to change devices.

We will now discuss the correcting effects of the drawing assist system against strong involuntary movements that occurred during the tulip tracing example. A participant' $\mathrm{s}$ drawing trajectory, including an 11 [s] span of strong involuntary movements, is shown on the xy-plane in Fig. 11; the change in position of each axis is shown in Fig. 12 , and the change in the velocity $V_{s}(i)$, drawing judgment velocity $V_{D}(i)$, and the attenuation weight coefficient $w(i)$ are shown in Fig. 13. A 9 [s] span of strong involuntary movements is marked with a colored dotted line. We can see that a wide range of hand movements occur with irregular direction, distance, and frequent veering. This means that the participant cannot trace the tulip example with voluntary movements or stop involuntary movements, causing them to lose drawing control. As shown in Fig. 13, the velocity $V_{s}(i)$ becomes higher than the transition velocity $v_{t h}=0.20[\mathrm{~m} / \mathrm{s}]$ and the attenuation weight coefficient $w(i)$ notably decreases during strong involuntary movements, while $V_{s}(i)$ remains less than $0.20[\mathrm{~m} / \mathrm{s}]$ and $w(i)$ does not approach zero after the strong involuntary movements have finished. It is confirmed that $\beta(i)$ does not attenuate voluntary movements, because the change in drawing judgment velocity is different from the change in the velocity and $w(i)$ becomes close to near zero when drawing judgment velocity values are low.

The AIBAF design includes the tuning of attenuation mentioned above by changing involuntary movements and drawing manipulations in real time to achieve drawing assistance in expressions that require the participant to perform continuous veering, even when involuntary movements occur. Additionally, the AIBAF design has properties to assist using window functions, such as color select and undo, even when involuntary movements occur.

Finally we discuss the AIBAF application range. (4) and (5) are effective in attenuating all types of nonperiodic involuntary movements losing control of the amount of exercise, not only athetosis. The upper limit $\alpha_{u l}$, the transition velocity $v_{t h}$, and the transition attenuation $D_{t h}$ are able to be tuned to the degree of involuntary movements of an individual.

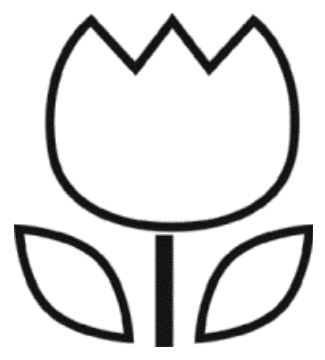

(a) Tracing design

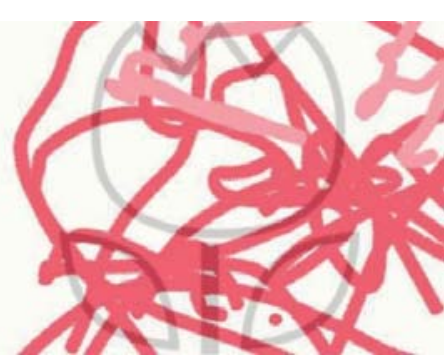

(b) Result with raw data

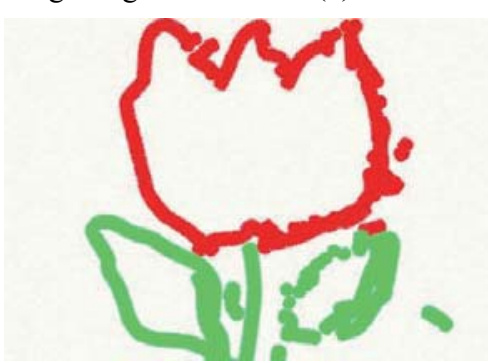

(c) Result with AIBAF

Fig. 10. Design and results of tulip tracing with raw and AIBAF 


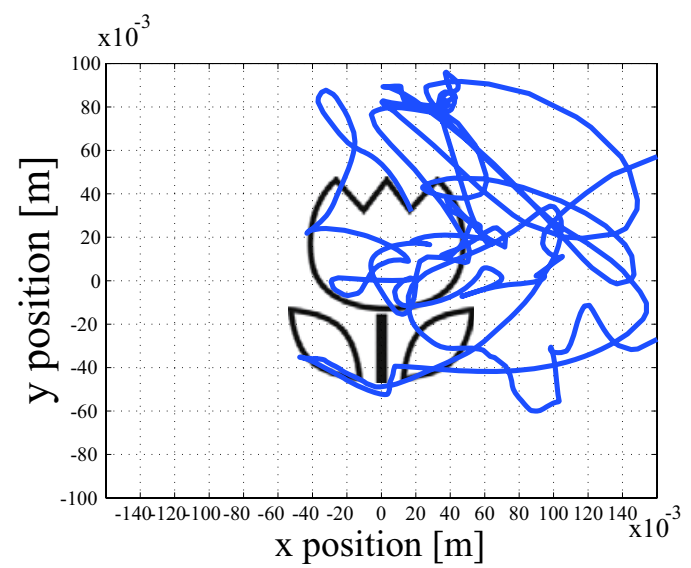

Fig. 11. Behavior of strong involuntary movement on $x-y$ plane during tulip tracing
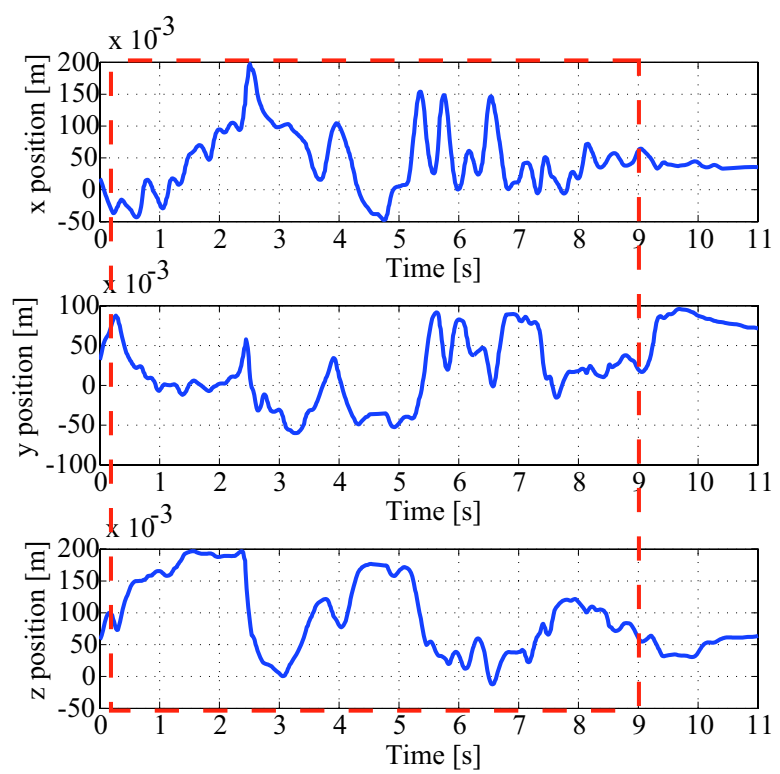

Fig. 12. Position change of strong involuntary movement during tulip tracing

(1) and (2), which are drawing judgment criteria, and $\beta(i)$, which is an attenuation coefficient for drawing judgment movements, are effective not only for patients with cerebral palsy or other physical disabilities, but also for unaffected individuals. Parameters of these equations are able to be tuned to the manipulation characteristics of individuals. The drawing assist system with AIBAF is effective in assisting drawing on a computer for individuals with nonperiodic involuntary movements that cause them to lose drawing control.

\section{CONCLUSION}

The AIBAF drawing assist system changes its attenuation to suit the involuntary movements and drawing movements of a user in real time. It is developed for users with athetoid cerebral palsy and muscle tension in order to analyze and correct involuntary movements during drawing. In drawing assist experiments using AIBAF the user could draw curves
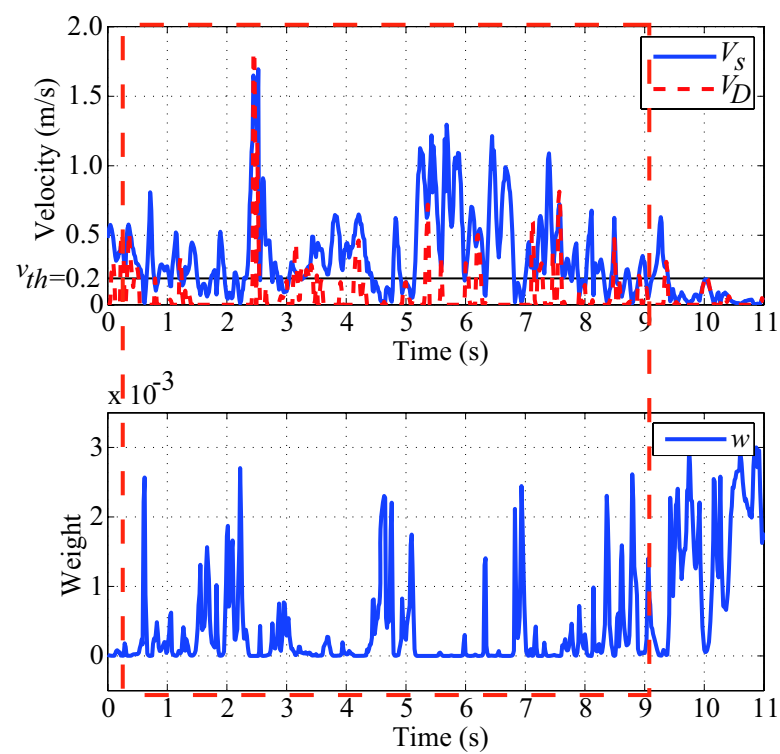

Fig. 13. Velocities and attenuation weight change of strong involuntary movement during tulip tracing

that require continuous veering, even when involuntary movements caused loss of drawing control.

\section{REFERENCES}

[1] J. L. Levine and M. A. Schappert, "A mouse adapter for people with hand tremor, IBM SYSTEMS JOURNAL," pp623-624, 2005

[2] D. Morimoto, M. Nawate, T. Watanabe, A. Abe, S. Fukuma and S. Honda, "A Painting Tool with Blurring Compensation for People Having Involuntary Hand Motion," Technical Report of The Institute of Erectronics, Information and Communication Engineers, pp59-64, 2004

[3] M. Shiraishi, H. Kawase and M. Shinya, "A Compensation Method for Hand-drawn Illustration by Moving Average," The Institute of Image Information and Television Engineers Technical Report, Vol.34, No.18, pp23-26, 2010

[4] H. Aoyama, T. Nakao, N. Miyagawa, N. Kubota, S. Horihata, and K. Yano, "Development of Drawing Assist System for Patients with Cerebral Palsy of the Tension Athetosis Type," Proceedings of IEEE ICRA2011 International Conference on Robotics and Automation, pp4664-4669, 2011

[5] R. Kaji, Diacrisis and Treatment of Involuntary Monement, pp72, 2006, Tokyo: SHINDAN-TO-CGIRYOSHA CO.

[6] S. Gomi, Rehabilitation Medicine Seminar (in Japanese), pp32, 2005, Ishiyaku Publishers, Inc.

[7] S. Nakai, "Power Grip Movement and Extension-Flexion Movement of Wrist in Cerebral Palsied Children," Japanese Journal of Special Education, pp7-15, 1984, The Japanese Association of Special Education

[8] N. Chino and N. Ando, Rehabilitation of Neurogenic Disorders, pp21, 2005, Tokyo: KANEHARA and CO.

[9] Ministry of Health, Labour and Welfare, Result of Survey on Children with Special Needs and Persons with Disabilities 2006 (in Japanese), Ministry of Health, Labour and Welfare, pp4, 2008

[10] Cabinet Office, Annual Report on Government Measures for Persons with Disabilities 2007, Cabinet Office, (2007), pp186

[11] PHANTOM Omni Haptic Device, available from 〈http://www.sensable.com/haptic-phantom-omni.htm〉, (accessed 2011-1-20)

[12] Art Rage, available from $\langle$ http://graphic.e-frontier.co.jp/artrage/ $\rangle$, (accessed 2011-9-13) 